\title{
DESENVOLVIMENTO DE DISPOSITIVO DIDÁTICO ALTERNATIVO DE BAIXO CUSTO PARA O ENSINO DO FENÔMENO DE PERMEABILIDADE
}

\author{
LOW-COST ALTERNATIVE DIDACTIC DEVICE DEVELOPMENT OF FOR PERMEABILITY \\ PHENOMENON TEACHING
}

Luís Felipe dos Santos Ribeiro ${ }^{1}$, André Augusto Nóbrega Dantas²

DOI: 10.37702/REE2236-0158.v40p202-208.2021

\begin{abstract}
RESUMO
Devido à atual crise financeira, que afeta a educação pública brasileira, faz-se necessário o uso de soluções criativas para que o ensino não seja afetado de forma negativa. O estudo de Engenharia, quando combinadas a teoria e a prática, fornece ao estudante uma grande eficácia no entendimento do conteúdo apresentado. Entretanto, no ramo das Ciências Tecnológicas, para se desenvolver a parte prática, é necessário utilizar equipamentos de laboratório e campo os quais, para serem obtidos, necessitam de um grande investimento por parte da Instituição. Dessa forma, este artigo apresenta a construção um permeâmetro de carga constante com materiais alternativos e de baixo custo. Esse dispositivo auxilia na explicação do fenômeno de permeabilidade dos solos, o qual pode ser a causa de inúmeros desastres ambientais. Dessa maneira, tem-se como objetivo auxiliar no ensino da disciplina de Geotecnia e promover uma melhor qualidade no ensino da Engenharia Civil do Instituto Federal de Educação, Ciência e Tecnologia de Goiás, Campus Formosa. O modelo final desenvolvido apresenta um baixíssimo custo quando comparado com o equipamento normatizado. Além disso, apresentou resultados satisfatórios para o coeficiente de permeabilidade dos solos testados, comprovando, assim, que o conhecimento técnico aliado à criatividade pode ser uma ferramenta eficiente em tempos difíceis para a pesquisa científica brasileira.
\end{abstract}

Palavras-chave: ensino; Engenharia Civil; Geotecnia; permeabilidade; dispositivo didático; sustentabilidade.

\begin{abstract}
Due to the current financial crisis affecting Brazilian public education, it is necessary to use creative solutions so that education is not affected. The study of engineering when combined theory and practice provides the student a great effectiveness in understanding the content covered. However, in the field of technological sciences, the practical part requires laboratory and field equipment that needs a large investment by the Institution to buy them. In this way, this article presents the development of permeameter of constant load with alternative and low-cost materials. This device helps to explain the phenomenon of soil permeability, which can be the cause of numerous environmental disasters. Thus, the objective of assisting in the teaching of the discipline of Geotechnics and promoting better quality in the teaching of Civil Engineering at the Instituto Federal de Educação, Ciência e Tecnologia de Goiás, Campus Formosa. The final model is cheaper than the original equipment, in addition, it presented satisfactory results for the tested soil. Thus proving that technical knowledge combined with creativity can be an efficient tool in difficult times for Brazilian scientific research.
\end{abstract}

Keywords: teaching; Civil Engineering; Geotechnics; permeability; didactic device; sustainability.

\footnotetext{
${ }^{1}$ Estudante (Graduação em andamento), Instituto Federal de Goiás, estudanteluis12@ gmail.com

2 Professor Dr., Instituto Federal de Goiás, eng.andreaugusto@yahoo.com.br
} 


\section{INTRODUÇÃO}

Durante o decorrer do curso de Bacharelado em Engenharia Civil os alunos se deparam com diversos conceitos teóricos os quais são de difícil compreensão sem o auxílio de uma abordagem prática. $\mathrm{O}$ processo de ensino tradicional e conservador está diretamente relacionado aos recursos didáticos utilizados pelo corpo docente em aulas presenciais. Com o passar do tempo e com a evolução dos métodos de ensino, tornou-se necessário que os docentes introduzam diferentes maneiras e processos didáticos para que os alunos compreendam de forma eficiente os conceitos de cada disciplina abordados em sala de aula.

O curso de Bacharelado em Engenharia Civil é dividido em áreas, sendo uma delas a Geotecnia. A primeira disciplina dessa área é Mecânica dos Solos, na qual um dos assuntos abordados é o estudo da influência da água nos solos, tópico de extrema importância para um profissional graduado em Engenharia Civil.

Com muita frequência, a água ocupa parte ou a totalidade dos vazios do solo. Esta, quando submetida a diferenças de potenciais, deslocase e pode vir a provocar colapsos e desastres geotécnicos (SOUSA PINTO, 2006). O conhecimento da percolação de água nos solos possibilita a análise de cálculo das vazões, análise de recalques e estudo da estabilidade que está relacionada às tensões efetivas, assim como a compreensão das causas de muitos problemas práticos ligados à percolação de água no solo, tais como: deslizamentos, piping entre outros.

A propriedade relacionada à capacidade de fluídos permearem no solo é a permeabilidade, indicada por meio do coeficiente de permeabilidade (k) (LAGACÉ, 2015). Esse coeficiente apresenta a velocidade na qual o fluído transita no interior do solo. Portanto, a velocidade será maior em situações em que há um maior número de vazios $\mathrm{e}$, consequentemente, teremos um maior $\mathrm{k}$ definindo a permeabilidade do solo. Entretanto, em situações em que o solo não apresenta grande número de vazios - solos compactados e solos de menor granulometria - são observadas baixas velocidades de percolação e um baixo $\mathrm{k}$.

$O$ índice de permeabilidade é uma propriedade fundamental para a determinação do solo que será adotado em diversas obras de terra. As barragens de terra, por exemplo, necessitam que o fluído seja mantido no seu interior; portanto, é fundamental a adoção de solos menos permeáveis. Porém, em muitos casos é feito um dreno interno (núcleo) e nesse momento é fundamental que se tenha um solo permeável. Então, o índice de permeabilidade se torna novamente essencial, pois é por meio deste que se torna possível controlar a vazão no interior da barragem.

De acordo com Burland (1987), o comportamento reológico dos solos só pode ser explicado de forma adequada mediante observações do material por meio de atividades em campo e laboratório. Sendo assim, a construção de modelos didáticos, capazes de demonstrar de forma prática os conceitos expostos em sala de aula, compõe uma ferramenta importante e, conforme afirmado por Steenfelt (2000), tal recurso deve ser sempre utilizado pelo corpo docente das Instituições no intuito de tornar o aprendizado mais eficiente.

Sendo assim, buscando tornar o curso de Engenharia Civil mais didático, são necessários equipamentos que promovam a união do conhecimento teórico ao ensino prático. O presente trabalho tem como objetivo apresentar o desenvolvimento de um dispositivo didático com uso de materiais de fácil acesso, reutilizáveis e de baixo custo. Este equipamento auxiliará o ensino da disciplina de Mecânica dos Solos, mais especificamente no estudo da permeabilidade dos solos: o permeâmetro de carga constante. A ideia de desenvolver o equipamento com materiais de fácil acesso, reutilizáveis e de baixo custo está ligada ao objetivo de facilitar a reprodução do equipamento em outras Instituições de Ensino Públicas, que também passam por problemas orçamentários devido à grave crise econômica enfrentada pelo país, fato que resulta na dificuldade de aquisição de equipamentos normatizados. 
Para a elaboração do dispositivo, foi feito um estudo de referências bibliográficas sobre o funcionamento do equipamento normatizado, com a finalidade de se chegar a um projeto que conciliasse ensino da teoria, recursos disponíveis e resultados satisfatórios após realização do ensaio para determinação do coeficiente de permeabilidade de solos.

\section{FUNDAMENTAÇÃO TEÓRICA}

\section{Permeabilidade}

A permeabilidade é uma propriedade inerente a todos os meios porosos, tendo grande relevância no estudo dos solos. Muitas substâncias naturais (rochas, madeiras, solos e ossos) e muitos materiais produzidos pelo homem (concretos e cerâmicas) podem ser classificados como materiais porosos. Dessa maneira, a compreensão do escoamento de fluidos através de meios porosos é de extrema importância para muitas áreas da engenharia, tais como: Geotecnia, Mecânica do Fluídos, Hidrologia entre outras.

A Lei de Darcy (Equação 1) apresenta o escoamento de um fluído em um meio poroso. A Lei é uma simplificação das equações de Navier e Stokes sob as hipóteses de escoamento laminar e incompressível. Além disso, ela permite determinar a velocidade e pressão que descrevem o fluxo de um fluido em um meio poroso:

$$
k=\frac{V \times L}{h \times A \times t}
$$

em que: $\mathrm{V}=$ Volume de fluído identificado no recipiente no tempo $\mathrm{t}\left(\mathrm{m}^{3}\right)$;

$\mathrm{h}=$ Altura da carga hidráulica, constante durante o ensaio $(\mathrm{m})$;

$\mathrm{A}=$ Área da secção do corpo de prova $\left(\mathrm{m}^{2}\right)$;

$\mathrm{L}=$ Altura do corpo de prova $(\mathrm{m})$;

$\mathrm{t}=$ Tempo decorrido para a água percolar no volume $\mathrm{V}(\mathrm{s})$.

Para determinar o coeficiente de permeabilidade também podem ser utilizadas leis empíricas, tal qual a Lei de Hazen (Equação 2). Esta correlaciona o coeficiente $\mathrm{k}$ ao diâmetro efetivo do solo ( $\left.D_{\text {ef etivo }}\right)$ obtido a partir do ensaio de granulometria:

$$
k=100 \times D_{\text {efet }}{ }^{2}
$$

Dessa maneira, em decorrência da heterogeneidade dos solos e do fato de o meio ser poroso, a determinação do coeficiente $\mathrm{k}$ com exatidão torna-se um procedimento complexo. Essa determinação, quando realizada em laboratório, é feita com o uso de equipamentos denominados permeâmetros (carga constate ou variável). Nesses ensaios são fundamentais várias repetições e a adoção de diversas amostras. Portanto, o maior empecilho para a realização destes é a falta de tal equipamento, em decorrência do seu alto valor.

\section{MATERIAIS E MÉTODOS}

O permeâmetro de carga constante desenvolvido foi construído no laboratório de Mecânica dos Solos do curso de Bacharelado em Engenharia Civil do Instituto Federal de Goiás - Campus Formosa. O equipamento foi construído com o uso de materiais reutilizáveis, a partir de uma análise minuciosa da norma ABNT: NBR 13292 - Determinação do Coeficiente de Permeabilidade de Solos Granulares a Carga Constante, com a finalidade de viabilização da construção de um modelo que suprisse o procedimento da normativa vigente. Já os materiais escolhidos deveriam satisfazer o objetivo do projeto: baixo custo, resultados satisfatórios, em concordância com a bibliografia pesquisada, e um recurso didático eficiente para a melhoria do aprendizado.

O equipamento foi construído com os seguintes materiais:

- galões (obsoletos) de água mineral de 20 litros;

- torneiras de filtro e pia;

- mangueira de nível;

- manta geotêxtil;

- brita 1 .

\section{Construção do equipamento}

Inicialmente, foi proposto um modelo que representasse o comportamento em uso do 
equipamento (Figura 1). Este seria então composto por dois recipientes (para armazenamento de água e corpo de prova, respectivamente), os quais seriam interligados por um tubo e dotados de objetos para controle da carga de água e medição da vazão percolada; para tal função, adotou-se o uso das torneiras.

\section{Figura 1 - Modelo do permeâmetro a ser desenvolvido}

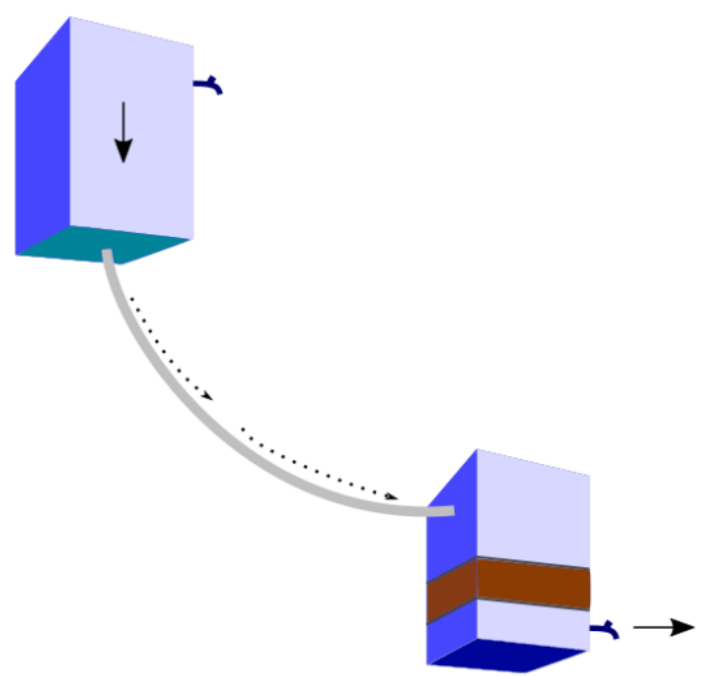

Fonte: acervo dos autores.

Assim, para a construção do equipamento foram confeccionados os recipientes com o uso de uma segueta e de um aparelho de solda. Com o corte dos recipientes foi possível obter uma maior trabalhabilidade dos mesmos e, posteriormente, com o uso do aparelho de solda, foram feitos quatro furos para fixação das torneiras.

Figura 2 - Torneiras fixadas

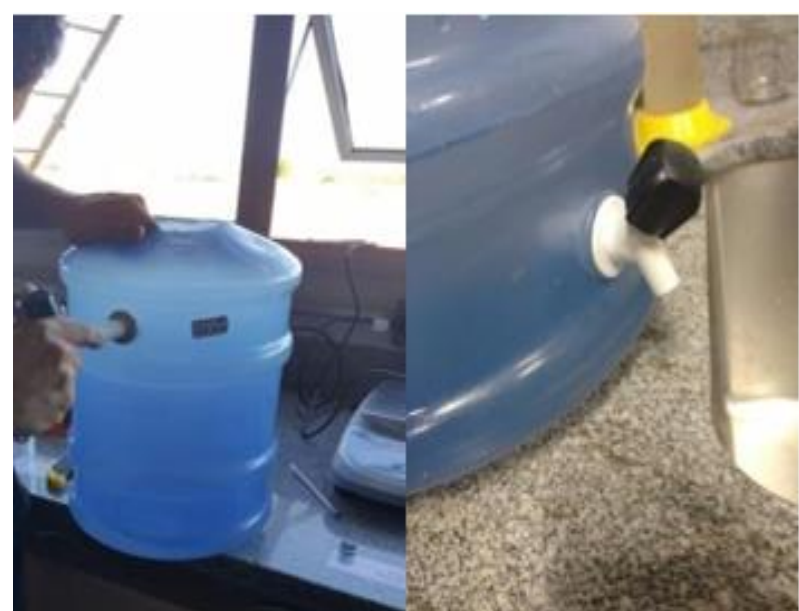

Após anexar as torneiras, utilizou-se uma mangueira de nível para unir os galões. Nesse momento foi necessário modificar o modelo inicial (Figura 1), pois, em decorrência da incompatibilidade entre a torneira de ligação dos galões e o corpo de prova, foi preciso fazer um furo na base do galão do corpo de prova e adotar a percolação vertical ascendente (Figura 3), uma opção presente na norma do ensaio.

Figura 3 - Representação gráfica final do galão com corpo de prova

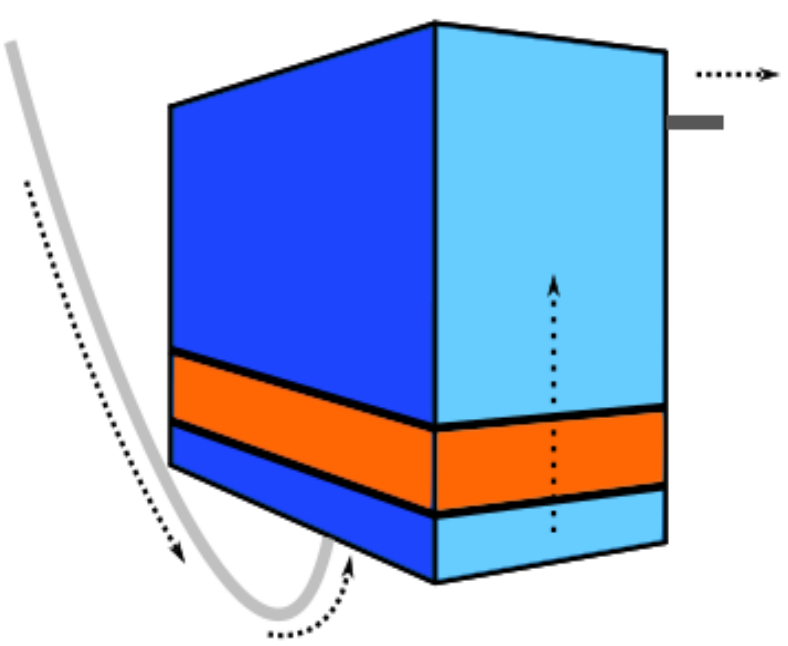

Fonte: acervo dos autores.

Feitos os furos, anexadas as torneiras e encaixada a mangueira, os galões estavam unidos e o equipamento estava pronto, conforme se apresenta na Figura 4.

Figura 4 - Equipamento pronto

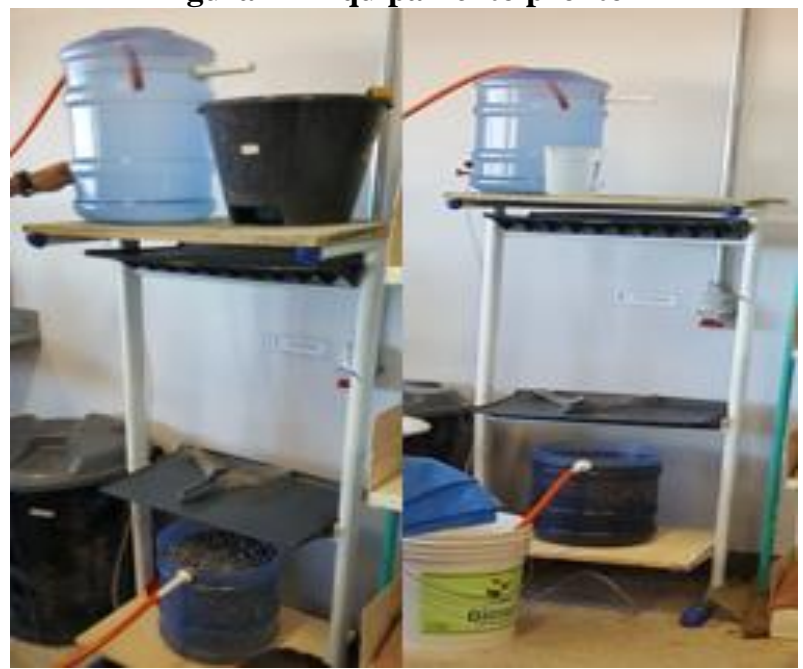

Fonte: acervo dos autores.

Fonte: acervo dos autores. 


\section{Realização do ensaio}

O enfoque principal na realização do ensaio é obter as variáveis necessárias, respeitando a normativa da Lei de Darcy para a determinação do coeficiente de permeabilidade. Dessa maneira, com o equipamento finalizado, partiu-se para a preparação do corpo de prova. $\mathrm{O}$ recipiente foi dividido em três camadas, cujo principal objetivo é uniformizar o fluxo por toda a seção transversal do corpo de prova. Conforme pode se verificar nas Figuras 5 e 6 , as camadas pré e pós corpo de prova são compostas por brita e dotadas de um geotêxtil (retalho que foi descartado) na interface de contato com o corpo de prova (camada central). A aplicação do geotêxtil também é essencial para manter as dimensões do corpo de prova que compôs a camada intermediária de forma que o mesmo permanecesse com suas dimensões constantes e recebesse um fluxo também constante de fluído.

\section{Figura 5 - Primeira camada e terceira camada} (Brita 1)

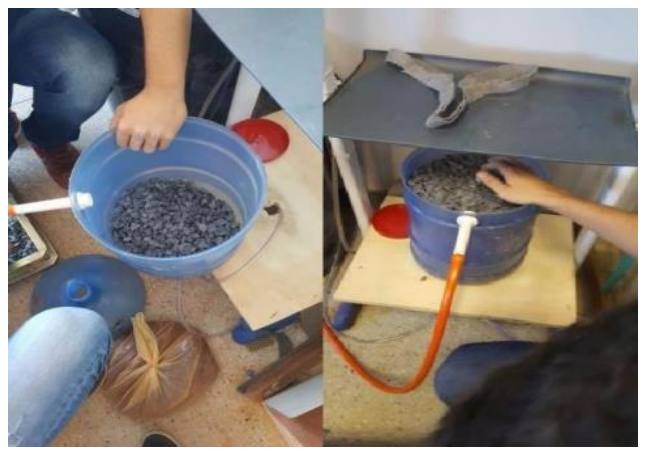

Fonte: acervo dos autores.

Figura 6 - Geossintético do tipo geotêxtil que separa o corpo de prova das demais cargas

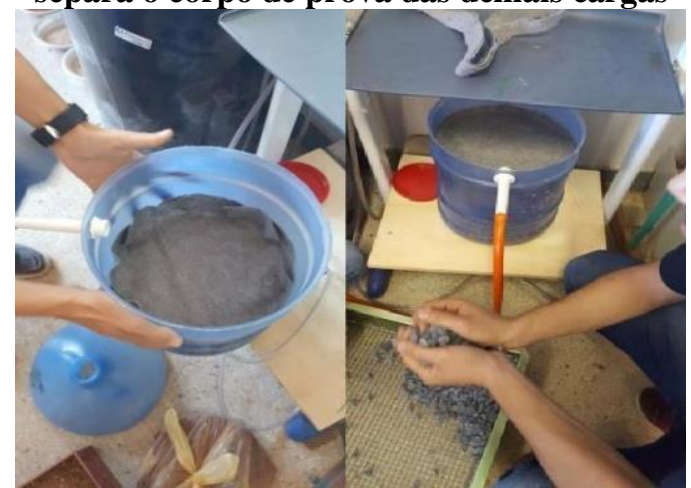

Fonte: acervo dos autores.

\section{RESULTADOS E DISCUSSÕES}

Após a construção do permeâmetro de carga constante, o equipamento foi verificado. Para tal, realizou-se a determinação do coeficiente de permeabilidade de uma areia média que se encontrava no Laboratório de Mecânica dos Solos do IFG - Campus Formosa. Comparouse resultados encontrados na bibliografia aos resultados obtidos pelo equipamento e pela Lei de Hazen. Os valores médios dos coeficientes de permeabilidade são apresentados na Tabela 1.

Tabela 1 - Tabela de coeficientes de permeabilidade médios

\begin{tabular}{|c|c|c|}
\hline Solos & Tipo de Solo & $\mathrm{k}(\mathrm{cm} / \mathrm{s})$ \\
\hline \multirow{2}{*}{ Permeáveis } & Pedregulho & $>10^{-3}$ \\
& Areias & $10^{-3} \mathrm{a} 10^{-5}$ \\
& Siltes & $10^{-5} \mathrm{a} 10^{-7}$ \\
\hline Impermeáveis & Argilas & $<10^{-7}$ \\
\hline
\end{tabular}

Fonte: Sousa Pinto, 2006 (adaptado).

\section{Lei de Hazen}

A primeira etapa consistiu na caracterização do solo quanto ao ensaio de granulometria, ABNT NBR 7181, pois, a partir deste, foi possível obter o Diâmetro Efetivo e o coeficiente $\mathrm{k}$ a partir da Lei de Hazen, conforme se apresenta na Tabela 2.

Figura 7 - Curva granulométrica da areia ensaiada

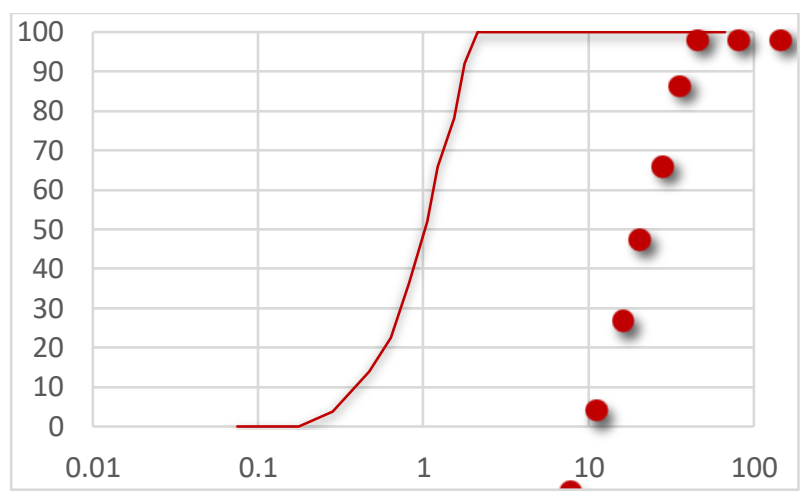

Fonte: elabora pelos autores.

$\mathrm{Na}$ curva, Figura 7, verifica-se a peneireira cuja porcentagem passante é de $10 \%$ como a de abertura 0.42 milímetros. 
Tabela 2 - Determinação do coeficiente $k$ a partir da Lei de Hazen

\begin{tabular}{|c|c|}
\hline $\operatorname{Defet}(\mathrm{mm})$ & $\mathrm{k}(\mathrm{cm} / \mathrm{s})$ \\
\hline 0,42 & $1,764 \times 10^{\wedge}-5$ \\
\hline
\end{tabular}

Fonte: elaborada pelos autores.

Assim, o resultado empírico obtido mostra concordância com o apresentado por Sousa Pinto, 2006.

\section{Permeâmetro alternativo}

O resultado obtido por meio do permeâmetro confeccionado, aliado à Lei de Darcy, é apresentado na Tabela 3.

Tabela 3 - Determinação do $k$ a partir do equipamento alternativo

\begin{tabular}{|c|c|}
\hline $\mathrm{t}(\mathrm{s})$ & 22,285 \\
$\mathrm{Q}\left(\mathrm{m}^{3} / \mathrm{s}\right)$ & $4,487 \times 10^{-5}$ \\
$\mathrm{~h}(\mathrm{~m})$ & 1,21 \\
$\mathrm{l}(\mathrm{m})$ & 0,11 \\
$\mathrm{As}\left(\mathrm{m}^{2}\right)$ & 0,05536 \\
$\mathrm{~V}\left(\mathrm{~m}^{3}\right)$ & 0,001 \\
\hline $\mathrm{k}(\mathrm{cm} / \mathrm{s})$ & $7,368 \times 10^{-5}$ \\
\hline
\end{tabular}

Fonte: elaborada pelos autores.

Novamente é possível observar a concordância entre $\mathrm{o}$ valor obtido em comparação ao valor médio apresentado na bibliografia. Dentro da ordem de grandeza característica de areias $\left(10^{-5}\right)$ é observada concordância entre os resultados obtidos para as leis de Darcy e Hazen; assim, com um maior número de ensaios, o equipamento alternativo pode se tornar uma alternativa não só para ensino, mas também para caracterização de diferentes solos.

\section{CONCLUSÕES}

Este trabalho foi realizado com o objetivo de melhorar o ensino das disciplinas de Geotecnia no Instituto Federal de Goiás, Campus Formosa, no qual o desenvolvimento de equipamentos didáticos alternativos é estimulado constantemente. Este projeto alia desenvolvimento da criatividade, sustentabilidade, melhoria da infraestrutura do laboratório de Mecânica dos Solos e otimização do aprendizado, uma vez que reúne teoria à prática.

Em relação ao equipamento desenvolvido, os resultados obtidos a partir das leis de Darcy e Hazen mostram que no futuro ele pode ser utilizado, além de ferramenta de ensino, como equipamento de caracterização. Portanto, há a demanda de um maior número de testes e ensaios.

A proposta do permeâmetro alternativo para fins didáticos é satisfeita, pois é possível visualizar os conceitos de permeabilidade dos solos, tais como a Lei de Darcy, linhas de fluxo e porosidade.

A respeito da questão financeira, apresentada em decorrência das limitações de verbas para o investimento em projetos de pesquisa e extensão, um dos objetivos do projeto foi tornar possível a construção de um equipamento didático com menor custo possível. Assim, além do baixo custo, destacase também o caráter de sustentabilidade, uma vez que são utilizados diversos materiais que estavam em desuso ou que seriam descartados.

\section{REFERÊNCIAS}

ABNT. ASSOCIAÇÃO BRASILEIRA DE NORMAS TECNICAS, NBR-7181 - Análise Granulométrica de Solos, outubro de 1984, Rio de Janeiro, 1984.

BURLAND, J. B. The Teaching of soil mechanics - a personal view. Proceedings... 9TH EUROPEAN CONF ON SOIL MECHANICS AND FOUNDATION ENGINEERING: GROUNDWATER EFFECTS IN GEOTECHNICAL ENGINEERING, p. 14271447, 1989.

\section{LAGACÉ, R. Conductivité hydraulique. Disponível em: <http://www.grr.ulaval.ca/gae_ 3001/Documents/Notes_2012/CH_06_K.pdf> . Acesso em: 15 nov. 2018.}

KNOP, A. et al. Sistema de Medição Digital de Permeabilidade de Solos. CIPPUS - Revista 
De Iniciação Científica, Canoas, v. 7, n. 1, 2019.

SOUSA PINTO, Carlos. Curso básico de Mecânica dos Solos, 16 Aulas. $3^{\text {a }}$. ed. São Paulo: Oficina de Textos, 2006.
STEENFELT, J. S. Teaching for the millennium or for the students? Proceedings... GEOENG GEOLOGICAL ENGINEERING, vol. 1, Melbourne, p. 826-840, 2000.
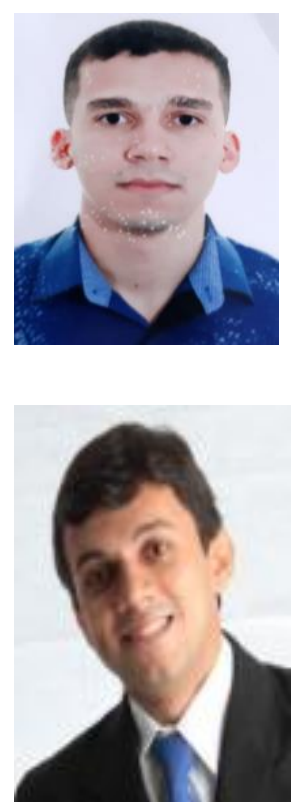

\section{DADOS BIOGRÁFICOS DOS AUTORES}

Luís Felipe dos Santos Ribeiro é Engenheiro Civil formado pelo Instituto Federal de Educação, Ciência e Tecnologia de Goiás. Atuou em três projetos de pesquisa como estudante de graduação nas áreas de Estruturas e Geotecnia, sendo este trabalho um dos equipamentos desenvolvidos ao longo de um projeto de pesquisa que durou dois anos. Realizou, como bolsista, intercâmbio acadêmico em Santiago do Chile pelo projeto LAPASSION (Latin-America Practices and Soft Skills for an Innovative Oriented Network) financiado pelo ERASMUS+. Atualmente é mestrando em Estruturas na Pontifícia Universidade Católica do Rio de Janeiro.

André Augusto Nóbrega Dantas é graduado em Engenharia Civil pela Universidade Federal do Rio Grande do Norte (2012). Possui mestrado em Engenharia Civil - Geotecnia também pela Universidade Federal do Rio Grande do Norte (2015) e doutorado em Geotecnia pela Universidade de Brasília. É professor efetivo do Instituto Federal de Educação, Ciência e Tecnologia de Goiás. Tem experiência na área de Engenharia Civil, com ênfase em Geologia de Engenharia, Geotecnia Ambiental, Geotecnia Aplicada à Mineração e Pavimentação, atuando principalmente nas seguintes áreas: barragens de rejeito, estabilidade, ensaios de laboratório, caracterização geotécnica e tecnológica de solos, resíduos industriais e de mineração, reciclagem e reaproveitamento de resíduos e gestão de pavimentos. 